\title{
The association between intake of dietary lycopene and other carotenoids and gestational diabetes mellitus risk during mid-trimester: a cross-sectional study
}

\author{
Qin $\mathrm{GaO}^{1}$, Chunrong Zhong ${ }^{1}$, Xuezhen Zhou ${ }^{1}$, Renjuan Chen ${ }^{1}$, Ting Xiong ${ }^{1}$, Miao Hong ${ }^{1}$, Qian $\mathrm{Li}^{1}$, \\ Man Kong ${ }^{2}$, Weizhen $\mathrm{Han}^{3}$, Guoqiang Sun ${ }^{4}$, Xuefeng Yang ${ }^{1}$, Nianhong Yang ${ }^{1 * \dagger}$ and Liping Hao ${ }^{1}+{ }^{*}$ \\ ${ }^{1}$ Department of Nutrition and Food Hygiene, Hubei Key Laboratory of Food Nutrition and Safety and the Ministry of Education \\ (MOE) Key Laboratory of Environment and Health, School of Public Health, Tongii Medical College, Huazhong University of \\ Science and Technology, 13 Hangkong Road, Wuhan 430030, People's Republic of China \\ ${ }^{2}$ Department of Medical Laboratory, The Central Hospital of Wuban, Wuban 430014, People's Republic of China \\ ${ }^{3}$ Department of Obstetrics and Gynecology, The Central Hospital of Wuhan, Wuban 430070, People's Republic of China \\ ${ }^{4}$ Department of Obstetrics and Gynecology, Hubei Maternal and Child Health Hospital, Wuban 430070, People's Republic of \\ China \\ (Submitted 13 March 2018 - Final revision received 28 August 2018 - Accepted 10 September 2018 - First published online 7June 2019)
}

\section{Abstract}

This study aimed to determine whether increased carotenoids intake was associated with reduced risk of gestational diabetes mellitus (GDM). We performed a cross-sectional analysis using data from Tongji Maternal and Child Health Cohort study. The dietary carotenoids intake of 1978 pregnant women was assessed using a researcher-administered FFQ before undertaking an oral glucose tolerance test at 24-28 weeks. Multivariate logistic and linear regression analyses were used to obtain the effect estimates. Participants in the highest quartile of lycopene intake showed a lower risk of GDM (OR 0.50; $95 \%$ CI 0.29, 0.86; $P_{\text {for trend }}=0 \cdot 007$ ) compared with those in the lowest quartile; each 1 mg increase in lycopene consumption was associated with a $5 \%\left(95 \%\right.$ CI 0.91, 0.99; $\left.P_{\text {for trend }}=0.020\right)$ decrease in GDM risk. No significant association was found between $\alpha$-carotene, $\beta$-carotene, $\beta$-cryptoxanthin, lutein/zeaxanthin intake and GDM risk. Multiple linear regression analysis suggested an inverse association between lycopene intake and fasting blood glucose (FBG) $\left(P_{\text {for trend }}<0.001\right)$; each 1 mg increase in lycopene intake was associated with 0.005 (95\% CI 0.002, 0.007; $\left.P_{\text {for trend }}<0.001\right) \mathrm{mmol} / \mathrm{l}$ decrease in FBG. Interaction analysis indicated consistent effect on each age or pre-BMI subgroup; however, a stronger protective effect of lycopene intake against GDM was observed among primigravid women (OR 0.20; $95 \%$ CI 0.07, 0.55 in the highest $v$. the lowest quartile of intake; $\left.P_{\text {for interaction }}=0.036\right)$. In conclusion, dietary lycopene intake was mainly assumed via reducing FBG to decrease GDM risk, and the protection was relatively increased among primigravid women.

Key words: Lycopene: Carotenoids: Gestational diabetes mellitus: Fasting blood glucose: Antioxidant activity

Gestational diabetes mellitus (GDM) is a form of hyperglycaemia that is first recognised during pregnancy. GDM is widely debated in view of steadily rising trend in case and constituting a major economic burden as it increased the risk for the offspring to develop obesity, as well as the risk for both mothers and children to develop type 2 diabetes mellitus later in life ${ }^{(1-4)}$. Although effective strategies to prevent GDM are mandatory, they are inadequate. Many factors have been shown to increase GDM risk, such as pre-pregnancy overweight or obesity, advanced maternal age, inadequate physical activity, multiparous status and firstdegree family history of diabetes ${ }^{(1,5-9)}$. Furthermore, epidemiological studies have found a link between oxidative stress and
$\mathrm{GDM}^{(10)}$, which indicated that dietary antioxidant nutrition may play a critical protective role against GDM development ${ }^{(11-13)}$.

Recently, carotenoids have received considerable interest due to their biological antioxidant activity in maintaining positive health ${ }^{(14,15)}$. Carotenoids, a group of natural fat-soluble pigments, are abundant in dark, green leafy and yellow-orange vegetables and fruits ${ }^{(16)}$. More than 600 carotenoids have so far been identified in nature, but six carotenoids ( $\alpha$-carotene, $\beta$-carotene, $\beta$-cryptoxanthin, lycopene and lutein/zeaxanthin) account for more than $95 \%$ of total blood carotenoids ${ }^{(15)}$.

Observational studies have suggested that carotenoids play a protective role in preventing chronic diseases, such as

Abbreviations: FBG, fasting blood glucose; GDM, gestational diabetes mellitus; IQR, interquartile range; OGTT, oral glucose tolerance test; PBG, postload blood glucose; TMCHC, Tongji Maternal and Child Health Cohort.

* Corresponding authors: Liping Hao, fax +86 27 83693307, email haolp@mails.tjmu.edu.cn; Nianhong Yang, fax +86 27 83650521, email zynh@mails.tjmu.edu.cn

$\dagger$ These authors contributed equally to this work. 
cancer, cardiovascular diseases and metabolic syndrome ${ }^{(17-19)}$. Meanwhile, large numbers of studies have shown the protective effect of carotenoids on diabetes risk $^{(20-22)}$, but the findings remained inconclusive. Randomised controlled trials with $\beta$-carotene supplementation consistently showed no effect on diabetes $^{(23-25)}$. A cross-sectional study showed an inverse relationship between dietary lycopene and fasting plasma glucose concentration $^{(26)}$, and three cohort studies found no association with diabetes among the general population or male smokers $^{(20,27,28)}$. The latest meta-analysis confirmed no significant association between lutein intake and diabetes risk ${ }^{(29)}$.

Considerable evidence indicates that GDM and type 2 diabetes share a common pathogenesis as insulin resistance or $\beta$-cell progressive dysfunction ${ }^{(30)}$. However, research on the effect of carotenoids on GDM is limited. We found a single case-control study that suggested no impact of $\beta$-carotene on $\mathrm{GDM}^{(31)}$. Thus, we aimed to investigate whether higher dietary carotenoid intake during mid-trimester pregnancy could reduce the risk of GDM.

\section{Methods \\ Study design and population}

The present study population was drawn from the Tongji Maternal and Child Health Cohort (TMCHC) study, a prospective cohort study conducted from September 2013 to May 2016 at three public hospitals in Wuhan, Hubei Province, China. The study aimed to determine the impact of maternal diet and lifestyle on pregnancy outcomes. The inclusion criteria were: (1) gestational age from 8 to 16 weeks, (2) maternal age $>18$ years, (3) no communication problem and (4) resident of Wuhan. Face-to-face interviews on the socioeconomic characteristics, dietary intake and lifestyles of the participants during each trimester were conducted by trained investigators. An oral glucose tolerance test (OGTT) was conducted at 24-28 weeks to diagnose GDM. All procedures performed in the present study were in accordance with the ethical standards of the Ethics Review Committee of Tongji Medical College of Huazhong University of Science and Technology (no. 201302). All participants provided informed written consent upon recruitment. This study was registered at clinicaltrials.gov as NCT03099837.

We collected the dietary data of 2750 participants by a researcher-administered semi-quantitative FFQ survey during 20-28 weeks, but subjects with unavailable dietary data (blank items $>10$ on FFQ: $n$, missing values for any vegetables or fruits: $n$ 170, extreme energy (<700 kcal $(<2930 \mathrm{~kJ})$ or $>5000 \mathrm{kcal}(>20920 \mathrm{~kJ})$ ): $n$ 2), unavailable OGTT data (missing OGTT data: $n$ 198, OGTT performed before FFQ: $n$ 321), multiple pregnancies ( $n$ 59) and pre-gestational diabetes ( $n$ 14) were excluded. Finally, the data of 1978 participants were obtained and analysed (online Supplementary Fig. S1).

\section{Dietary assessment}

Dietary data were collected using an FFQ before OGTT was conducted. The developed FFQ consisted of sixty-one food items, which were assembled into ten food groups: cereals, meat, fish, eggs, beans, vegetables, fruits, milk and milk products, nuts and beverages. The vegetable and fruit food groups were used to assess the dietary carotenoid intake. The vegetable group mainly included dark green leafy vegetables, light leafy vegetables, carrots and pumpkins, fresh beans, melons and solanaceous vegetables, including tomatoes and peppers. The fruit group mainly included citrus fruits, pomes, bananas, berries, watermelons and mangoes ${ }^{(32)}$. Participants were asked to report their usual food consumption during the past 4 weeks, including the type of food, frequency and amount. Daily energy and nutrient consumption (except for carotenoids) were calculated using a dietary software based on the China Food Composition Database ${ }^{(33)}$; carotenoids intake was calculated based on the United States Department of Agriculture Nutrient Database $^{(34)}$. The FFQ was a reasonably reliable and valid tool to investigate the diet of urban participants, as the reproducibility and validity coefficients of food and nutrition were all statistically significant ${ }^{(32)}$. The correlation coefficients between two FFQ were 0.34 for $\alpha$-carotene, 0.37 for $\beta$-carotene, 0.38 for $\beta$-cryptoxanthin, 0.36 for lycopene and 0.31 for lutein/ zeaxanthin. The de-attenuated correlation coefficients between the average of two FFQ and three $24 \mathrm{~h}$ recalls were 0.33 for $\alpha$-carotene, 0.37 for $\beta$-carotene, 0.41 for $\beta$-cryptoxanthin, 0.45 for lycopene and 0.40 for lutein/zeaxanthin (online Supplementary Tables S1 and S2).

\section{Assessment of other covariates}

We collected information on socioeconomic characteristics (age, ethnicity, education and income level, gravidity, parity and family history of diabetes), and anthropometric data including height, pre-pregnancy weight, weight at enrolment and weight at OGTT screening, systolic blood pressure and diastolic blood pressure at enrolment, and lifestyle habits such as smoking status and alcohol consumption before pregnancy, physical activity, sleep status and dietary supplements use during mid-trimester. Pre-pregnancy BMI (pre-BMI) was calculated as pre-pregnancy weight divided by height squared $\left(\mathrm{kg} / \mathrm{m}^{2}\right)$. Pre-pregnancy weight was self-reported, and height was measured at the first antenatal visit. If pre-pregnancy weight was missing, it was substituted with the enrolment weight. We examined two weight gain rate patterns before OGTT screening: the first trimester weight gain rate was measured as the enrolment weight minus pre-pregnancy weight and divided by the gestational age at enrolment; the second trimester weight gain rate was measured as the OGTT screening weight minus the enrolment weight and divided by the gestational age at OGTT screening minus gestational age at enrolment. If the OGTT screening weight was missing, it was replaced with the weight closest to the screening and no more than 3 weeks before or 1 week after the screening. Sleep time referred to the duration of night time sleep during pregnancy. Maternal sleep quality during pregnancy was classified as 'never', 'occasionally', 'sometimes' and 'often insomniac'; poor sleep quality referred to those participants who selected the answer 'often insomniac'. No participants used pure carotenoids supplementation in our study, so we recorded the use of compound supplements containing vitamin $\mathrm{A}$, vitamin $\mathrm{C}$ or vitamin E during mid-pregnancy. 


\section{Oral glucose tolerance test assessment}

All participants received routine screening by means of $75 \mathrm{~g}$ 2-h OGTT after at least $8 \mathrm{~h}$ overnight fast at 24-28 gestational weeks. Fasting blood glucose (FBG), 1-h postload blood glucose (PBG) and 2-h PBG concentration were collected from the laboratory test report. The International Association of Diabetes and Pregnancy Study Groups (IADPSG) recommends GDM to be diagnosed if one or more of the following criteria are met: FBG concentration $\geq 5.1 \mathrm{mmol} / \mathrm{l}$, 1 -h $\mathrm{PBG}$ concentration $\geq 10.0 \mathrm{mmol} / \mathrm{l}$ and $2-\mathrm{h}$ PBG concentration $\geq 8.5 \mathrm{mmol} / \mathrm{l}^{(35)}$.

\section{Statistical analysis}

Demographic characteristics were described as means for parametrically distributed data, medians for non-parametrically distributed data and percentages for categorical data. Characteristics among quartile groups of carotenoids were compared using ANOVA for normally distributed variables, or the Kruskal-Wallis rank test for non-normally distributed variables, and the $\chi^{2}$ test for categorical variables. All dietary nutrients and carotenoids intake were energy adjusted using the residual method ${ }^{(36)}$.

We demonstrated the correlation between dietary carotenoids intake and GDM prevalence by scatterplot smoothing. Binary logistic regression analysis was applied to evaluate the OR of GDM among different dietary carotenoids intake quartiles and per unit (1 mg) increment of dietary carotenoids intake. Linear trends were evaluated by creating a continuous variable for carotenoids using the median value of each quartile. Furthermore, multivariate linear regression analysis was adopted to demonstrate the relationship between lycopene consumption and FBG, 1-h and 2-h PBG concentration, and the results were presented as $\beta$ with $95 \%$ CI. We then demonstrated the association of carotenoids and GDM risk in subgroups including the age group ( $\leq 28$ years or $>28$ years), pre-BMI group $\left(<24.0 \mathrm{~kg} / \mathrm{m}^{2}\right.$ or $\left.\geq 24 \mathrm{~kg} / \mathrm{m}^{2}\right)$ and gravidity group $(1$ or $\geq 2)$. The interactions between these stratification variables and carotenoids intake were tested using the multivariate logistic regression model, and the likelihood ratio test was used to assess the statistical significance of the interaction terms. All analyses used the same covariates, with some of them reported as common confounders or GDM factors ${ }^{(1,5-9,11,12,37,38)}$, and were those changed the effect estimate over $10 \%$ for corresponding models. In addition, the season of FFQ was adjusted to account for the variation in diet for different seasons. The first model was adjusted for maternal age, pre-BMI, first trimester weight gain rate, second trimester weight gain rate, systolic blood pressure, diastolic blood pressure, gravidity, parity, family history of diabetes, smoking and alcohol use status, physical activity and poor sleep quality. The second model was further adjusted for the gestational age of FFQ, season of FFQ and dietary intake of vitamin $\mathrm{C}$, vitamin $\mathrm{E}$, fibre, cholesterol, retinol, Se, $\mathrm{Zn}, \mathrm{Mg}, \mathrm{Fe}$ and $\mathrm{Cu}$ intake, which were all adjusted for energy.

All data were analysed using statistical packages $\mathrm{R}$ (The R Foundation; http://www.r-project.org; v. 3.1.2) and Empower(R) (http://www.empowerstats.com; X\&Y Solutions Inc.). All tests were conducted assuming a two-sided alternative hypothesis and $P<0.05$ was considered statistically significant.

\section{Results}

In the present study, 1978 participants were included for analysis (age: $28.1 \pm 3.5$ years and pre-BMI: $20.7 \pm 2.6 \mathrm{~kg} / \mathrm{m}^{2}$ ) and 152 $(7.7 \%)$ were diagnosed GDM. Median intakes were as follows: $\alpha$-carotene, 0.277 (interquartile range (IQR) $0.135,0.650$ ) $\mathrm{mg} / \mathrm{d}$, $\beta$-carotene, 3.091 (IQR 2.089, 4.566) $\mathrm{mg} / \mathrm{d}, \beta$-cryptoxanthin, 0.293 (IQR 0.156, 0.477) mg/d, lycopene 2.082 (IQR 0.145, 5.266 ) $\mathrm{mg} / \mathrm{d}$ and lutein/zeaxanthin, 2.499 (IQR 1.572, $3 \cdot 803) \mathrm{mg} / \mathrm{d}$. Table 1 shows the distribution of socio-economic, anthropometric and lifestyle characteristics and dietary intake of the sample according to quartiles of lycopene intake. The characteristics of other carotenoids are shown in online Supplementary Table S3. Subjects with higher lycopene intake had higher vitamin $\mathrm{C}, \mathrm{Mg}, \mathrm{Fe}$ and $\mathrm{Cu}$ consumption, as well as lower second trimester weight gain rate, GDM prevalence and FBG.

The associations between carotenoids intake and GDM prevalence by scatterplot smoothing were displayed in online Supplementary Fig. S2. An inverse correlation was observed between lycopene intake and GDM risk $(P=0 \cdot 032)$ after adjusting for covariates. OR for GDM risk according to quartiles of carotenoids consumption are shown in Table 2. In model 1 logistic regression analyses, we found that the OR for the highest $v$. the lowest quartiles of $\beta$-cryptoxanthin and lycopene were $0.61\left(95 \%\right.$ CI $\left.0.38,0.98 ; P_{\text {for trend }}=0.084\right)$ and 0.50 (95\% CI 0.30, 0.84; $\left.P_{\text {for trend }}=0 \cdot 005\right)$, respectively. After further adjusting for potential confounders, the protective effect of $\beta$-cryptoxanthin was attenuated (OR 0.65; $95 \%$ CI 0.35, 1.21; $\left.P_{\text {for trend }}=0.278\right)$, but the effect of lycopene was still significant (OR 0.50; $95 \%$ CI 0.29, 0.86; $P_{\text {for trend }}=0.007$ ); each $1 \mathrm{mg}$ increase in lycopene consumption was associated with $5 \%$ (OR 0.95; $95 \%$ CI 0.91, 0.99; $\left.P_{\text {for trend }}=0.020\right)$ decrease in GDM risk. However, no significant effect of $\alpha$-carotene, $\beta$-carotene and lutein/zeaxanthin on GDM risk was found in our research.

Table 3 presents further multivariate linear regression analysis to examine the impact of lycopene consumption on blood glucose concentration. Compared with the lowest lycopene intake quartile, the $\beta$-coefficients across quartiles of lycopene intake with FBG were -0.032 (95\% CI -0.074, 0.011), -0.105 (95\% CI $-0.148,-0.062)$ and -0.119 (95\% CI -0.163 , $-0.075)\left(P_{\text {for trend }}<0.001\right)$ after adjusting for potential confounders; each $1 \mathrm{mg}$ increase in lycopene intake was associated with 0.005 (95\% CI 0.002, 0.007) mmol/1 decrease in FBG concentration $(P<0.001)$. No significant associations were observed between lycopene intake and 1-h or 2-h PBG concentration.

Stratified analysis was used to examine potential modification, according to age, pre-BMI and gravidity strata (Fig. 1). The protective effect of lycopene against GDM was consistent for each age and pre-BMI subgroups. However, the primigravid women was found to exhibit enhanced protection (OR 0.20; $95 \%$ CI $0.07,0.55$ in the highest $v$. the lowest quartile of intake; $\left.P_{\text {for interaction }}=0 \cdot 036\right)$.

\section{Discussion}

In this large cross-sectional study, we established that dietary lycopene intake, rather than the intake of other carotenoids, was 
Table 1. Basic characteristics of study subjects by quartiles $(Q)$ of dietary lycopene intake from the Tongji Maternal and Children Health Cohort (TMCHC) study (Mean values, median values; numbers of participants; percentages)

\begin{tabular}{|c|c|c|c|c|c|}
\hline \multirow[b]{2}{*}{ Characteristics } & \multicolumn{4}{|c|}{ Lycopene } & \multirow[b]{2}{*}{$P^{*}$} \\
\hline & Q1 & Q2 & Q3 & Q4 & \\
\hline$n$ & 495 & 494 & 493 & 496 & \\
\hline Median intake (mg/d) & 0.015 & $1 \cdot 281$ & 3.185 & 8.992 & $<0.001$ \\
\hline \multicolumn{6}{|l|}{ Socio-economic factors } \\
\hline Age (years) & $28 \cdot 1$ & $28 \cdot 1$ & $28 \cdot 1$ & $28 \cdot 2$ & 0.987 \\
\hline Han ethnicity (\%) & $99 \cdot 2$ & $97 \cdot 0$ & $95 \cdot 9$ & 98.0 & 0.008 \\
\hline Education ( $\geq 16$ years, \%) & 55.4 & $62 \cdot 2$ & $60 \cdot 1$ & 57.9 & 0.261 \\
\hline Income ( $\geq 5000 \mathrm{CNY}, \%)$ & $59 \cdot 0$ & 64.8 & $62 \cdot 9$ & $57 \cdot 1$ & 0.117 \\
\hline \multicolumn{6}{|c|}{ Anthropometry and lifestyle factors } \\
\hline pre-BMI $\left(\mathrm{kg} / \mathrm{m}^{2}\right)$ & $20 \cdot 8$ & $20 \cdot 7$ & $20 \cdot 7$ & $20 \cdot 7$ & 0.906 \\
\hline GAE (weeks) & $13 \cdot 2$ & $12 \cdot 6$ & $13 \cdot 0$ & 12.5 & 0.004 \\
\hline FWGR (kg/week) & 0.08 & 0.09 & 0.08 & 0.08 & 0.951 \\
\hline SWGR (kg/week) & 0.56 & 0.55 & 0.52 & 0.48 & $<0.001$ \\
\hline $\mathrm{SBP}(\mathrm{mmHg})$ & $112 \cdot 7$ & 113.1 & $114 \cdot 6$ & 113.4 & 0.047 \\
\hline $\mathrm{DBP}(\mathrm{mmHg})$ & $70 \cdot 4$ & $70 \cdot 8$ & $70 \cdot 8$ & 69.9 & 0.253 \\
\hline Primigravid (\%) & $56 \cdot 0$ & $59 \cdot 3$ & $59 \cdot 8$ & 57.5 & 0.588 \\
\hline Primiparity (\%) & $86 \cdot 1$ & $85 \cdot 8$ & 85.4 & $82 \cdot 3$ & 0.302 \\
\hline FHD (\%) & 9.5 & 8.9 & 7.9 & $8 \cdot 7$ & 0.850 \\
\hline Smoker (\%) & 13.9 & $12 \cdot 6$ & $12 \cdot 6$ & $12 \cdot 7$ & 0.492 \\
\hline Alcohol use (\%) & $10 \cdot 1$ & $10 \cdot 3$ & 13.4 & $15 \cdot 9$ & 0.056 \\
\hline PA (\%) & $32 \cdot 1$ & $32 \cdot 8$ & $31 \cdot 2$ & $29 \cdot 4$ & 0.939 \\
\hline PSQ (\%) & 8.7 & 4.7 & 3.4 & $5 \cdot 6$ & 0.003 \\
\hline \multicolumn{6}{|l|}{ Dietary intake $\dagger$} \\
\hline Season ('Sum + Aut', \%) & $41 \cdot 6$ & 44.5 & 53.5 & 67.9 & $<0.001$ \\
\hline GAF (weeks) & $24 \cdot 1$ & 23.5 & 23.4 & $24 \cdot 0$ & 0.045 \\
\hline Energy (kJ/d) & 8019.9 & $9165 \cdot 9$ & $8706 \cdot 1$ & 8488.5 & $<0.001$ \\
\hline Total fat $(\mathrm{g} / \mathrm{d})$ & 76.7 & 74.6 & 74.8 & 69.5 & $<0.001$ \\
\hline Vitamin C $(\mathrm{mg} / \mathrm{d})$ & $152 \cdot 4$ & 166.9 & 174.5 & 189.7 & $<0.001$ \\
\hline Vitamin E (mg/d) & 48.8 & 48.8 & 48.5 & 48.1 & 0.650 \\
\hline Fibre $(\mathrm{mg} / \mathrm{d})$ & 14.5 & $15 \cdot 3$ & $15 \cdot 1$ & $15 \cdot 8$ & $<0.001$ \\
\hline Cholesterol (mg/d) & $376 \cdot 2$ & $365 \cdot 1$ & 379.2 & $360 \cdot 8$ & 0.389 \\
\hline Retinol $(\mu \mathrm{g} / \mathrm{d})$ & $200 \cdot 9$ & 181.1 & 193.5 & $186 \cdot 3$ & 0.183 \\
\hline $\operatorname{Se}(\mu \mathrm{g} / \mathrm{d})$ & $42 \cdot 4$ & $42 \cdot 3$ & $42 \cdot 7$ & $42 \cdot 2$ & 0.341 \\
\hline $\mathrm{Zn}(\mathrm{mg} / \mathrm{d})$ & $10 \cdot 3$ & $10 \cdot 2$ & $10 \cdot 3$ & $10 \cdot 4$ & 0.255 \\
\hline$M g(m g / d)$ & 321.3 & 331.7 & $333 \cdot 3$ & 346.8 & $<0.001$ \\
\hline $\mathrm{Fe}(\mathrm{mg} / \mathrm{d})$ & $18 \cdot 2$ & $18 \cdot 7$ & $19 \cdot 1$ & 19.8 & $<0.001$ \\
\hline $\mathrm{Cu}(\mathrm{mg} / \mathrm{d})$ & $2 \cdot 2$ & 2.4 & 2.5 & 2.6 & $<0.001$ \\
\hline Supplement use (\%) & 38.2 & 40.5 & $41 \cdot 0$ & 38.7 & 0.906 \\
\hline \multicolumn{6}{|l|}{ OGTT } \\
\hline GAO (weeks) & $26 \cdot 2$ & $26 \cdot 1$ & $26 \cdot 2$ & $26 \cdot 2$ & 0.862 \\
\hline GDM (\%) & $9 \cdot 3$ & 8.9 & $7 \cdot 7$ & 4.8 & 0.037 \\
\hline $\mathrm{FBG}(\mathrm{mmol} / \mathrm{l})$ & 4.46 & 4.42 & 4.35 & 4.32 & $<0.001$ \\
\hline 1-h PBG (mmol/l) & 7.40 & 7.48 & 7.42 & 7.47 & 0.765 \\
\hline 2-h PBG (mmol/l) & $6 \cdot 27$ & $6 \cdot 38$ & $6 \cdot 33$ & $6 \cdot 29$ & 0.358 \\
\hline
\end{tabular}

pre-BMI, pre-pregnancy BMI; GAE, gestational age at enrolment; FWGR, first trimester weight gain rate; SWGR, second trimester weight gain rate; SBP, systolic blood pressure; DBP, diastolic blood pressure; FHD, family history of diabetes; PA, physical activity; PSQ, poor sleep quality; Sum + Aut, summer and autumn; GAF, gestational age at FFQ survey; OGTT, oral glucose tolerance test; GAO, gestational age at OGTT screening; GDM, gestational diabetes mellitus; FBG, fasting blood glucose; PBG, postload blood glucose.

*ANOVA or $\chi^{2}$ test or the Kruskal-Wallis rank test was used as appropriate.

† Values of dietary intake were medians and were energy-adjusted by residual methods (except for energy)

inversely associated with GDM risk after adjusting for a wide range of potentially confounding factors. Furthermore, the protection was mainly assumed due to reducing FBG, instead of $1-h$ and 2-h PBG. Notably, the observed protective effect was stronger in primigravid than in multiple pregnancies women.

To the best of our knowledge, no large-scale study has demonstrated the association between carotenoids consumption and GDM risk. In fact, carotenoids, the marker of fruits and vegetables intake, has less been focused on maternal health. As aforementioned, only one case-control study ( $n$ 80) revealed no intergroup difference of $\beta$-carotene intake between GDM and non-GDM groups $^{(31)}$, which was consistent with our results. Another nested case-control study confirmed the protective effect of serum lutein and zeaxanthin on maternal health, but the outcome was preeclampsia ${ }^{(39)}$. We found no significant association between dietary $\alpha$-carotene, $\beta$-carotene, $\beta$-cryptoxanthin and lutein/zeaxanthin intake and GDM risk, which was consistent with most previously mentioned studies on type 2 diabetes ${ }^{(23-26,29)}$.

Our finding that lycopene intake was significantly associated with a reduced risk of GDM was supported by previous studies. Based on reported studies, a daily lycopene intake of 5-7 mg in normal healthy human may be sufficient to maintain circulating levels, combat oxidative stress and prevent chronic diseases ${ }^{(40)}$. In this study, the median of lycopene intake in the highest quartile was $8.992 \mathrm{mg} / \mathrm{d}$, which indicated the intake may be enough for pregnant women to prevent GDM. Intriguingly, 
Table 2. Association of dietary carotenoids intake with gestational diabetes mellitus (GDM) risk (Odds ratios and $95 \%$ confidence intervals)

\begin{tabular}{|c|c|c|c|c|c|c|c|}
\hline \multirow[b]{2}{*}{ Carotenoids } & & \multirow[b]{2}{*}{ Total $n$} & \multirow[b]{2}{*}{ Cases } & \multicolumn{2}{|c|}{ Model 1* } & \multicolumn{2}{|c|}{ Model $2 \dagger$} \\
\hline & & & & OR & $95 \% \mathrm{Cl}$ & OR & $95 \% \mathrm{Cl}$ \\
\hline \multirow[t]{7}{*}{$\alpha$-Carotene } & Q1 $(\leq 0.135)$ & 495 & 40 & 1.00 & Ref. & 1.00 & Ref. \\
\hline & Q2 (0.136-0.279) & 494 & 37 & 0.98 & $0.61,1.57$ & 1.09 & $0.65,1.83$ \\
\hline & Q3 (0.280-0.649) & 494 & 35 & 0.91 & $0.56,1.47$ & 0.98 & $0.58,1.64$ \\
\hline & Q4 $(\geq 0.650)$ & 495 & 40 & 0.97 & $0.61,1.55$ & 1.06 & $0.63,1.76$ \\
\hline & $P_{\text {for trend }}$ & & - & 0.931 & & 0.941 & \\
\hline & Per unit & & - & 1.01 & $0.80,1.28$ & 1.03 & $0.80,1.31$ \\
\hline & $P$ & & - & 0.950 & & 0.968 & \\
\hline \multirow[t]{7}{*}{$\beta$-Carotene } & Q1 ( $\leq 2 \cdot 089)$ & 495 & 44 & 1.00 & Ref. & 1.00 & Ref. \\
\hline & Q2 (2.090-3.089) & 494 & 36 & 0.77 & $0.48,1.23$ & 0.79 & $0.48,1.28$ \\
\hline & Q3 (3.090-4.562) & 494 & 31 & 0.65 & $0.40,1.06$ & 0.67 & $0.38,1.15$ \\
\hline & Q4 ( $\geq 4.563)$ & 495 & 41 & 0.85 & $0.54,1.34$ & 0.92 & $0.47,1.80$ \\
\hline & $P_{\text {for trend }}$ & & - & 0.578 & & 0.855 & \\
\hline & Per unit & & - & 1.01 & $0.94,1.10$ & $1 \cdot 10$ & $0.96,1.26$ \\
\hline & $P$ & & - & 0.725 & & 0.733 & \\
\hline \multirow[t]{7}{*}{$\beta$-Cryptoxanthin } & Q1 $(\leq 0 \cdot 155)$ & 495 & 51 & 1.00 & Ref. & 1.00 & Ref. \\
\hline & Q2 (0.156-0.292) & 494 & 33 & 0.64 & $0.40,1.03$ & 0.65 & $0.40,1.06$ \\
\hline & Q3 (0.293-0.477) & 494 & 38 & 0.74 & $0.47,1.17$ & 0.76 & $0.46,1.26$ \\
\hline & Q4 $(\geq 0.478)$ & 495 & 30 & 0.61 & $0.38,0.98$ & 0.65 & $0.35,1.21$ \\
\hline & $P_{\text {for trend }}$ & & - & 0.084 & & 0.278 & \\
\hline & Per unit & & - & 0.60 & $0.30,1.20$ & 0.76 & $0.30,1.95$ \\
\hline & $P$ & & - & 0.150 & & 0.132 & \\
\hline \multirow[t]{7}{*}{ Lycopene } & Q1 $(\leq 0.145)$ & 495 & 46 & 1.00 & Ref. & 1.00 & Ref. \\
\hline & Q2 (0.146-2.082) & 494 & 44 & 0.97 & $0.62,1.51$ & 0.97 & $0.62,1.51$ \\
\hline & Q3 (2.083-5.264) & 493 & 38 & 0.83 & $0.52,1.32$ & 0.82 & $0.51,1.32$ \\
\hline & Q4 $(\geq 5 \cdot 265)$ & 496 & 24 & 0.50 & $0.30,0.84$ & 0.50 & $0.29,0.86$ \\
\hline & $P_{\text {for trend }}$ & & - & 0.005 & & 0.007 & \\
\hline & Per unit & & - & 0.95 & $0.91,0.99$ & 0.95 & $0.91,0.99$ \\
\hline & $P$ & & - & 0.018 & & 0.020 & \\
\hline \multirow[t]{7}{*}{ Lutein/zeaxanthin } & Q1 $(\leq 1.571)$ & 495 & 40 & 1.00 & Ref. & 1.00 & Ref. \\
\hline & Q2 (1.572-2.499) & 494 & 35 & 0.83 & $0.51,1.34$ & 0.79 & $0.49,1.30$ \\
\hline & Q3 (2.500-3.802) & 494 & 30 & 0.71 & $0.43,1.17$ & 0.70 & $0.41,1.19$ \\
\hline & Q4 $(\geq 3.803)$ & 495 & 47 & 1.04 & $0.66,1.64$ & 1.08 & $0.58,2.00$ \\
\hline & $P_{\text {for trend }}$ & & - & 0.718 & & 0.373 & \\
\hline & Per unit & & - & 1.05 & $0.96,1.14$ & $1 \cdot 13$ & $0.97,1.33$ \\
\hline & $P$ & & - & 0.290 & & 0.120 & \\
\hline
\end{tabular}

Q, quartile; Ref., reference; pre-BMI, pre-pregnancy BMI; FWGR, first trimester weight gain rate; SWGR, second trimester weight gain rate; SBP, systolic blood pressure; DBP, diastolic blood pressure; FHD, family history of diabetes; PA, physical activity; PSQ, poor sleep quality; GAF, gestational age at FFQ survey; Sum + Aut, summer and autumn.

* Model 1 was adjusted for maternal age, pre-BMI, FWGR, SWGR, SBP, DBP, gravidity ( 1 as reference), parity ( 0 as reference), FHD ('no' as reference), smoking status ('no' as reference), alcohol use status ('no' as reference), PA ('yes' as reference), PSQ ('no' as reference).

† Model 2 was further adjusted for GAF, season of FFQ ('Sum + Aut' as reference), supplement use ('no' as reference), as well as for vitamin C, vitamin E, fibre, cholesterol, retinol, Se, $\mathrm{Zn}, \mathrm{Mg}, \mathrm{Fe}$ and $\mathrm{Cu}$ intake, which were all adjusted for energy.

Table 3. Association between dietary lycopene intake and blood glucose

( $\beta$-Coefficients and $95 \%$ confidence intervals)

\begin{tabular}{|c|c|c|c|c|c|c|c|c|c|c|c|}
\hline & \multirow{2}{*}{$\begin{array}{c}\text { Q1 } \\
\beta\end{array}$} & \multicolumn{2}{|r|}{ Q2 } & \multicolumn{2}{|r|}{ Q3 } & \multicolumn{2}{|r|}{ Q4 } & \multirow[b]{2}{*}{$P_{\text {for trend }}$} & \multicolumn{2}{|r|}{ Per unit } & \multirow[b]{2}{*}{$P$} \\
\hline & & $\beta$ & $95 \% \mathrm{Cl}$ & $\beta$ & $95 \% \mathrm{Cl}$ & $\beta$ & $95 \% \mathrm{Cl}$ & & $\beta$ & $95 \% \mathrm{Cl}$ & \\
\hline \multicolumn{12}{|l|}{ FBG } \\
\hline Model $1^{*}$ & 0 & -0.027 & $-0.070,0.015$ & $-0 \cdot 101$ & $-0.144,-0.058$ & -0.117 & $-0.160,-0.074$ & $<0.001$ & -0.005 & $-0.008,-0.003$ & $<0.001$ \\
\hline $\begin{array}{l}\text { Model } 2 \dagger \\
1-\mathrm{h} \text { PBG }\end{array}$ & 0 & -0.032 & $-0.074,0.011$ & $-0 \cdot 105$ & $-0.148,-0.062$ & -0.119 & $-0.163,-0.075$ & $<0.001$ & -0.005 & $-0.007,-0.002$ & $<0.001$ \\
\hline Model $1^{*}$ & 0 & 0.086 & $-0.091,0.263$ & 0.009 & $-0.169,0.187$ & 0.080 & $-0.098,0.257$ & 0.539 & 0.005 & $-0.005,0.016$ & 0.326 \\
\hline $\begin{array}{l}\text { Model 2† } \\
\text { 2-h PBG }\end{array}$ & 0 & 0.084 & $-0.093,0.261$ & -0.016 & $-0 \cdot 195,0 \cdot 164$ & 0.038 & $-0.147,0.223$ & 0.920 & 0.004 & $-0.007,0.015$ & 0.528 \\
\hline Model 1* & 0 & 0.123 & $-0.011,0.257$ & 0.062 & -0.073 & 0.028 & $-0.107,0$ & & 0.0 & & \\
\hline Model $2 \dagger$ & 0 & 0.112 & $-0.023,0.246$ & 0.049 & $-0.087,0.186$ & 0.007 & $-0.134,0.147$ & 0.550 & 0.003 & $-0.005,0.012$ & 0.435 \\
\hline
\end{tabular}

Q, quartile; FBG, fasting blood glucose; PBG, postload blood glucose; pre-BMI, pre-pregnancy BMI; FWGR, first trimester weight gain rate; SWGR, second trimester weight gain rate; SBP, systolic blood pressure; DBP, diastolic blood pressure; FHD, family history of diabetes; PA, physical activity; PSQ, poor sleep quality; GAF, gestational age at FFQ survey; Sum + Aut, summer and autumn.

* Model 1 was adjusted for maternal age, pre-BMI, FWGR, SWGR, SBP, DBP, gravidity ( 1 as reference), parity ( 0 as reference), FHD ('no' as reference), smoking status ('no' as reference), alcohol use status ('no' as reference), PA ('yes' as reference), PSQ ('no' as reference).

† Model 2 was further adjusted for GAF, season of FFQ ('Sum + Aut' as reference), supplement use ('no' as reference), as well as for vitamin C, vitamin E, fibre, cholesterol, retinol, Se, $\mathrm{Zn}, \mathrm{Mg}, \mathrm{Fe}$ and $\mathrm{Cu}$ intake, which were all adjusted for energy. 


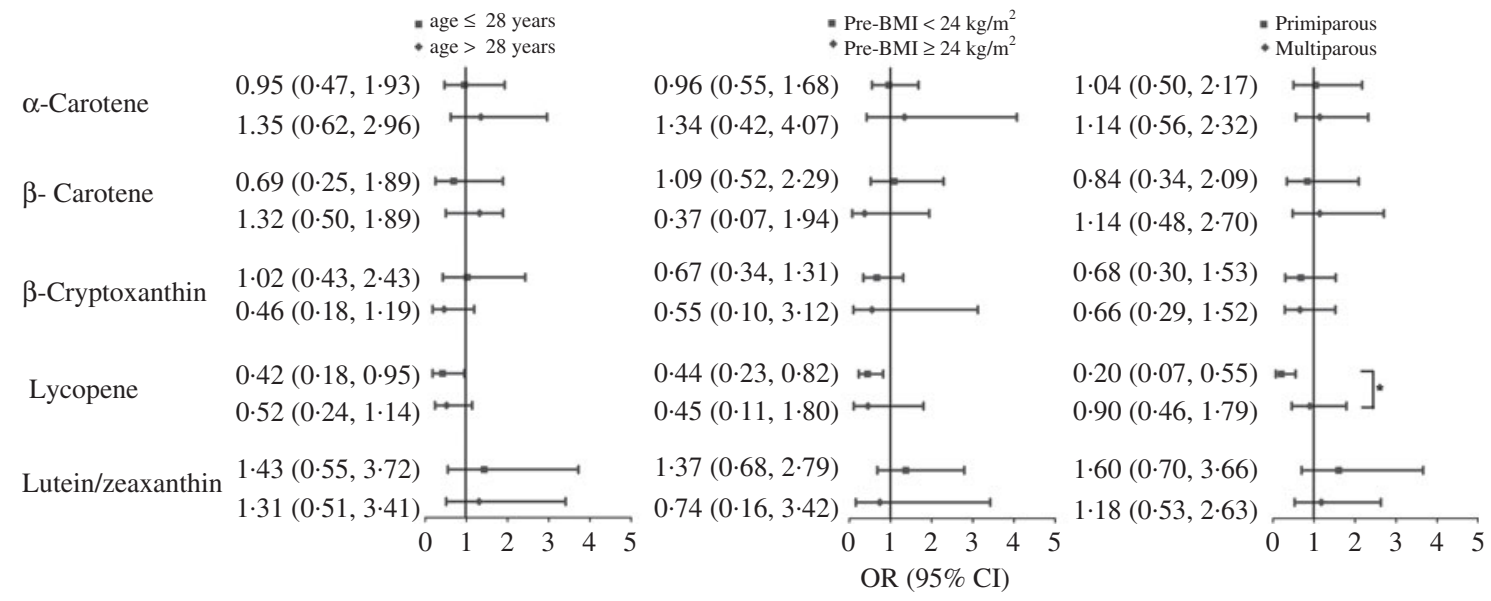

Fig. 1. Multivariate-adjusted $\mathrm{OR}(95 \% \mathrm{Cl})$ of gestational diabetes mellitus by age, pre-pregnancy BMI (pre-BMI) and gravidity, comparing quartile 4 with quartile 1 of dietary intake of carotenoids. Model adjusted for maternal age, pre-BMI, first trimester weight gain rate, second trimester weight gain rate, systolic blood pressure, diastolic blood pressure, gravidity ( 1 as reference), parity ( 0 as reference), family history of diabetes ('no' as reference), smoking status ('no' as reference), alcohol use status ('no' as reference), physical activity ('yes' as reference), poor sleep quality ('no' as reference) and further adjusted for gestational age at FFQ survey, season of FFQ ('summer + autumn' as reference), supplement use ('no' as reference), as well as for vitamin C, vitamin E, fibre, cholesterol, retinol, selenium, zinc, magnesium, iron and copper intake, which were all adjusted for energy. ${ }^{*} P_{\text {for interaction }}<0.05$.

the protection was mainly manifested via reducing FBG, instead of 1-h or 2-h PBG, which was in line with the previous study. The cross-sectional study of Ylonen et al. suggested that a higher lycopene intake was related to lower FBG concentration ${ }^{(26)}$. By contrast, a multi-centre study proposed that serum lycopene was inversely related to PBG concentration ${ }^{(41)}$. However, many studies have found no relationship between lycopene and diabetes, which maybe due to differences in study design, population, range of carotenoids and sample size. For instance, one nested case-control study confirmed a crude inverse relationship between plasma lycopene and type 2 diabetes, but the effect was attenuated after adjusting for potential confounding factors $^{(42)}$.

Although the mechanism of the association between dietary lycopene intake and GDM risk has yet not to be clarified, several biological properties of lycopene may account for its protective effect. Lycopene, mainly found in tomato, tomato products and watermelon, possesses more potent antioxidative activity than $\alpha$ - or $\beta$-carotene ${ }^{(43,44)}$. Given the association between oxidative stress and prevalence and development of GDM, the benefit of lycopene against oxidative damage is indispensable ${ }^{(44,45)}$. Indeed, insulin resistance due to progressive $\beta$-cell dysfunction has also been recognised as a fundamental predictor for GDM development ${ }^{(46,47)}$. High hepatic insulin resistance is generally manifested by overproduction of glucose in the basal state despite fasting hyperinsulinaemia ${ }^{(48)}$. Intriguingly, animal experiments showed that lycopene ameliorated insulin resistance by inhibiting the pathogenesis of hepatic steatosis and improving the lipid profiles ${ }^{(49,50)}$. Beyond that, lycopene may induce $\beta$-cell intercellular communication through gap junctions $^{(51)}$, which contributed to the control of insulin secretion and glucose tolerance ${ }^{(52)}$. All these properties potentially explained the regulation of FBG by higher dietary lycopene consumption.

However, the lack of association between other carotenoids and GDM risk cannot be explained. Of all carotenoids addressed in this study, $\beta$-carotene is known to be a strong antioxidant. In our analysis the increase in $\beta$-carotene intake has been associated with the decrease in GDM risk; however, the association was not statistically significant. One possible reason is that lycopene possesses more potent antioxidative activity ${ }^{(43,44)}$. In addition, the intake of $\beta$-carotene was narrower than the intake of lycopene in this study. The narrow intake of $\beta$-carotene may be difficult to find the significant effect on GDM risk. All the above may partly explain why $\beta$-carotene exerted no effect on GDM risk. We also found no association between $\alpha$-carotene and GDM risk, which could possibly be explained by the high correlation between $\beta$-carotene and $\alpha$-carotene. In our analysis, the correlation coefficient between $\alpha$-carotene and $\beta$-carotene was 0.535 (data were not shown). Meanwhile, no significant effect of lutein/zeaxanthin on GDM risk was found in this study, which was consistent with the result of lutein/zeaxanthin on type 2 diabetes in a meta-analysis ${ }^{(29)}$.

Notably, this study provided new evidence that the protection of lycopene against GDM was significantly stronger in primigravid women, and several possible mechanisms might account for this result. Pregnancy itself could be regarded as a state of increased insulin resistance, and recurrent pregnancies may result in an additive effect ${ }^{(53)}$. Moreover, previous research demonstrated the increased pre-BMI and complicated metabolic changes among multiparous women, which may increase GDM risk ${ }^{(9,54)}$. The protection of lycopene against GDM may be overwhelmed by complicated pro-oxidant activity generated by multiple pregnancies. However, the potential mechanism remains poorly understood and should be elucidated by further animal and epidemiological research.

The main strength of our study is that the dietary survey was conducted via face-to-face interview using a validated FFQ. The researcher-administered FFQ survey was considered as the proper approach, while a self-administered FFQ survey was not advisable in China. Furthermore, the large size of the study sample, different categories of carotenoids, high quality of data 
and adjustment for a large numbers of potential confounding variables are also the major strength.

Nevertheless, there are some limitations to our study. First, based on a cross-sectional study design, we cannot draw a conclusion about causality. However, the main strength of this study might reduce the risk of report bias and recall bias as the participants had not known their GDM status when FFQ survey was conducted. Second, although numerous confounding factors were adjusted, we could not exclude uncontrolled factors, such as dietary lipid content, which may influence the bioaccessibility and bioavailability of carotenoids ${ }^{(55)}$. However, we pronounced carotenoids-independent effect by adjusting for dietary vitamin $\mathrm{C}$ and vitamin $\mathrm{E}$ intake, which maybe the main confounding factors and negatively related with GDM risk ${ }^{(11,12)}$. Finally, despite the large sample size of the study, the number of GDM cases was relatively small for stratified analyses. However, all GDM cases in our study were strictly diagnosed based on FBG, as well as 1-h and 2-h postload glucose and according to new diagnostic criteria, rather than directly copying from hospitalised medical records. In fact, the prevalence of GDM in the present study $(7.7 \%)$ was lower than the incidence from medical records ( $8.2 \%$ ) (data not shown). We admitted that we might have missed some cases of GDM rather than erroneously considered a non-GDM participant as a GDM case. Of course, the potential bias would most likely attenuate our results, and we would have a higher statistical power if we had more cases of GDM.

In conclusion, the findings from this large cross-sectional study investigating the associations between carotenoids consumption and GDM risk suggest that dietary lycopene intake is associated with a lower risk of GDM in a Chinese population, particularly among primigravid women. This work has important implications for public health with respect to the prevention of GDM. Further and larger studies should be conducted to confirm our findings and to elucidate the underlying mechanisms in the future.

\section{Supplementary material}

For supplementary material/s referred to in this article, please visit https://doi.org/10.1017/S0007114519000606

\section{Acknowledgements}

The authors are indebted to all the pregnant women included in the TMCHC study for their continued cooperation and participation. The authors also express their thanks to the staff of The Central Hospital of Wuhan, Hubei Maternal and Child Health Hospital and Jiang'an Maternal and Child Health Hospital for their considerable assistance with many aspects of this study. The authors gratefully acknowledge everyone in the TMCHC study group.

The TMCHC study was supported by the National Program on Basic Research Project of China (N. Y., no. 2013FY114200) and the National Natural Science Foundation of China (L. H., no. 81573149).

The authors' responsibilities were as follows: Q. G. collected data, analysed the data and drafted the manuscript; C. Z., X. Z.,
R. C., T. X., M. H. and Q. L. collected data and contributed to the discussion and reviewed/edited the manuscript; M. K., W. H. and G. S. coordinated data collection in the field; L. H., X. Y. and N. Y. participated study design and reviewed and edited the manuscript; N. Y. is the guarantor of this study, who had full access to all the data in the study and took responsibility for the integrity of the data and the accuracy of the data analysis; all the authors read and approved the final manuscript.

All the authors declare that they have no conflict of interest relevant to the content of this article.

\section{References}

1. Reece EA, Leguizamon G \& Wiznitzer A (2009) Gestational diabetes: the need for a common ground. Lancet 373, 17891797.

2. Boney CM, Verma A, Tucker R, et al. (2005) Metabolic syndrome in childhood: association with birth weight, maternal obesity, and gestational diabetes mellitus. Pediatrics 115, e290-e296.

3. Dabelea D, Mayer-Davis EJ, Lamichhane AP, et al. (2008) Association of intrauterine exposure to maternal diabetes and obesity with type 2 diabetes in youth: the SEARCH CaseControl Study. Diabetes Care 31, 1422-1426.

4. Bellamy L, Casas JP, Hingorani AD, et al. (2009) Type 2 diabetes mellitus after gestational diabetes: a systematic review and meta-analysis. Lancet 373, 1773-1779.

5. Catalano PM, McIntyre HD, Cruickshank JK, et al. (2012) The hyperglycemia and adverse pregnancy outcome study: associations of GDM and obesity with pregnancy outcomes. Diabetes Care 35, 780-786.

6. Savvidou M, Nelson SM, Makgoba M, et al. (2010) First-trimester prediction of gestational diabetes mellitus: examining the potential of combining maternal characteristics and laboratory measures. Diabetes 59, 3017-3022.

7. Agha-Jaffar R, Oliver N, Johnston D, et al. (2016) Gestational diabetes mellitus: does an effective prevention strategy exist? Nat Rev Endocrinol 12, 533-546.

8. Badon SE, Enquobahrie DA, Wartko PD, et al. (2017) Healthy lifestyle during early pregnancy and risk of gestational diabetes mellitus. Am J Epidemiol 186, 326-333.

9. Boudet-Berquier J, Salanave B, Desenclos JC, et al. (2017) Sociodemographic factors and pregnancy outcomes associated with prepregnancy obesity: effect modification of parity in the nationwide Epifane birth-cohort. BMC Pregnancy Childbirth 17, 273.

10. Coughlan MT, Vervaart PP, Permezel M, et al. (2004) Altered placental oxidative stress status in gestational diabetes mellitus. Placenta 25, 78-84.

11. Zhang C, Williams MA, Sorensen TK, et al. (2004) Maternal plasma ascorbic acid (vitamin C) and risk of gestational diabetes mellitus. Epidemiology 15, 597-604.

12. Ley SH, Hanley AJ, Sermer M, et al. (2013) Lower dietary vitamin $\mathrm{E}$ intake during the second trimester is associated with insulin resistance and hyperglycemia later in pregnancy. Eur J Clin Nutr 67, 1154-1156.

13. Bo S, Lezo A, Menato G, et al. (2005) Gestational hyperglycemia, zinc, selenium, and antioxidant vitamins. Nutrition 21, 186-191.

14. Zielinska MA, Wesolowska A, Pawlus B, et al. (2017) Health effects of carotenoids during pregnancy and lactation. Nutrients 9, 838.

15. Fiedor J \& Burda K (2014) Potential role of carotenoids as antioxidants in human health and disease. Nutrients 6, 466-488. 
16. Couillard C, Lemieux S, Vohl MC, et al. (2016) Carotenoids as biomarkers of fruit and vegetable intake in men and women. $\mathrm{Br}$ J Nutr 116, 1206-1215.

17. Zhou Y, Wang T, Meng Q, et al. (2016) Association of carotenoids with risk of gastric cancer: a meta-analysis. Clin Nutr $\mathbf{3 5}$, 109-116.

18. Petyaev IM (2016) Lycopene deficiency in ageing and cardiovascular disease. Oxid Med Cell Longev 2016, 3218605.

19. Liu J, Shi WQ, Cao Y, et al. (2014) Higher serum carotenoid concentrations associated with a lower prevalence of the metabolic syndrome in middle-aged and elderly Chinese adults. Br J Nutr 112, 2041-2048.

20. Montonen J, Knekt P, Jarvinen R, et al. (2004) Dietary antioxidant intake and risk of type 2 diabetes. Diabetes Care 27, 5 .

21. Sluijs I, Cadier E, Beulens JW, et al. (2015) Dietary intake of carotenoids and risk of type 2 diabetes. Nutr Metab Cardiovasc Dis 25, 376-381.

22. Akbaraly TN, Fontbonne A, Favier A, et al. (2008) Plasma carotenoids and onset of dysglycemia in an elderly population: results of the epidemiology of vascular ageing study. Diabetes Care 31, 1355-1359.

23. Song Y, Cook NR, Albert CM, et al. (2009) Effects of vitamins C and $\mathrm{E}$ and beta-carotene on the risk of type 2 diabetes in women at high risk of cardiovascular disease: a randomized controlled trial. Am J Clin Nutr 90, 429-437.

24. Liu S, Ajani U, Chae C, et al. (1999) Long-term beta-carotene supplementation and risk of type 2 diabetes mellitus: a randomized controlled trial. JAMA 282, 1073-1075.

25. Kataja-Tuomola M, Sundell JR, Mannisto S, et al. (2008) Effect of alpha-tocopherol and beta-carotene supplementation on the incidence of type 2 diabetes. Diabetologia 51, 47-53.

26. Ylonen K, Alfthan G, Groop L, et al. (2003) Dietary intakes and plasma concentrations of carotenoids and tocopherols in relation to glucose metabolism in subjects at high risk of type 2 diabetes: the Botnia Dietary Study. Am J Clin Nutr 77, 1434-1441.

27. Wang L, Liu S, Manson JE, et al. (2006) The consumption of lycopene and tomato-based food products is not associated with the risk of type 2 diabetes in women. J Nutr 136, 620-625.

28. Kataja-Tuomola MK, Kontto JP, Mannisto S, et al. (2011) Intake of antioxidants and risk of type 2 diabetes in a cohort of male smokers. Eur J Clin Nutr 65, 590-597.

29. Leermakers ET, Darweesh SK, Baena CP, et al. (2016) The effects of lutein on cardiometabolic health across the life course: a systematic review and meta-analysis. Am J Clin Nutr 103, 481-494.

30. Law KP \& Zhang H (2017) The pathogenesis and pathophysiology of gestational diabetes mellitus: deductions from a threepart longitudinal metabolomics study in China. Clin Chim Acta 468, 60-70

31. Parast VM \& Paknahad Z (2017) Antioxidant status and risk of gestational diabetes mellitus: a case-control study. Clin Nutr Res 6, 81-88.

32. Zhang H, Qiu X, Zhong C, et al. (2015) Reproducibility and relative validity of a semi-quantitative food frequency questionnaire for Chinese pregnant women. Nutr J 14, 56.

33. Yang Y (2004) China Food Composition. Beijing: Peking University Medical Press.

34. United States Department of Agriculture and Agricultural Research Service (2017) USDA Food Composition Databases. https://ndb.nal.usda.gov/ndb/nutrients/index (accessed October 2017).
35. International Association of Diabetes, Pregnancy Study Groups Consensus Panel, Metzger BE, et al. (2010) International Association of Diabetes and Pregnancy Study Groups Recommendations on the diagnosis and classification of hyperglycemia in pregnancy. Diabetes Care 33, 676-682.

36. Willett W \& Stampfer MJ (1986) Total energy intake: implications for epidemiologic analyses. Am J Epidemiol 124, 17-27.

37. MacDonald SC, Bodnar LM, Himes KP, et al. (2017) Patterns of gestational weight gain in early pregnancy and risk of gestational diabetes mellitus. Epidemiology 28, 419-427.

38. Zhang C \& Rawal S (2017) Dietary iron intake, iron status, and gestational diabetes. Am J Clin Nutr 106, 1672s-1680s.

39. Cohen JM, Kramer MS, Platt RW, et al. (2015) The association between maternal antioxidant levels in midpregnancy and preeclampsia. Am J Obstet Gynecol 213, 695.e1-695.e13.

40. Rao AV \& Shen H (2002) Effect of low dose lycopene intake on lycopene bioavailability and oxidative stress. Nutr Res $\mathbf{2 2}$, $1125-1131$

41. Coyne T, Ibiebele TI, Baade PD, et al. (2005) Diabetes mellitus and serum carotenoids: findings of a populationbased study in Queensland, Australia. Am J Clin Nutr 82, 685-693.

42. Wang L, Liu S, Pradhan AD, et al. (2006) Plasma lycopene, other carotenoids, and the risk of type 2 diabetes in women. $A m J$ Epidemiol 164, 576-585.

43. Di Mascio P, Kaiser S \& Sies H (1989) Lycopene as the most efficient biological carotenoid singlet oxygen quencher. Arch Biochem Biophys 274, 532-538.

44. Wang XD (2012) Lycopene metabolism and its biological significance. Am J Clin Nutr 96, 1214S-1222S.

45. Cocate PG, Natali AJ, Alfenas RC, et al. (2015) Carotenoid consumption is related to lower lipid oxidation and DNA damage in middle-aged men. Br J Nutr 114, 257-264.

46. Buchanan TA \& Xiang AH (2005) Gestational diabetes mellitus. J Clin Invest 115, 485-491.

47. Buchanan TA, Xiang A, Kjos SL, et al. (2007) What is gestational diabetes? Diabetes Care 30, S105-S111.

48. Vangipurapu J, Stancakova A, Kuulasmaa T, et al. (2011) A novel surrogate index for hepatic insulin resistance. Diabetologia 54, 540-543.

49. Ahn J, Lee H, Jung CH, et al. (2012) Lycopene inhibits hepatic steatosis microRNA-21-induced downregulation of fatty acidbinding protein 7 in mice fed a high-fat diet. Mol Nutr Food Res 56, 1665-1674.

50. Zeng Z, He W, Jia Z, et al. (2017) Lycopene improves insulin sensitivity through inhibition of STAT3/Srebp-1c-mediated lipid accumulation and inflammation in mice fed a high-fat diet. Exp Clin Endocrinol Diabetes 125, 610-617.

51. Stahl W \& Sies H (1996) Lycopene: a biologically important carotenoid for humans? Arch Biochem Biophys 336, 1-9.

52. Charollais A, Gjinovci A, Huarte J, et al. (2000) Junctional communication of pancreatic beta cells contributes to the control of insulin secretion and glucose tolerance. J Clin Invest 106, 235-243

53. Zhang X, Shu XO, Gao YT, et al. (2009) Pregnancy, childrearing, and risk of stroke in Chinese women. Stroke 40, 2680-2684.

54. Koski-Rahikkala H, Pouta A, Pietilainen K, et al. (2006) Does parity affect mortality among parous women? J Epidemiol Community Health 60, 968-973.

55. Kim JE, Gordon SL, Ferruzzi MG, et al. (2015) Effects of egg consumption on carotenoid absorption from co-consumed, raw vegetables. Am J Clin Nutr 102, 75-83. 